\title{
Preparation and training: the key to better patient involvement
}

Articles which compare practical examples of different methods of involving users are rare, so the paper by van Wersch and Eccles ${ }^{1}$ in this issue of Quality in Health Care is of particular interest. It examines four methods of involving patients in the development of clinical guidelines which, in itself, is an area that has received relatively little attention. The four methods tested were:

- inclusion of individual patients in guideline development groups;

- "one off" meeting with patients;

- series of workshops with patients;

- inclusion of a patient advocate in guideline development groups.

The findings in this specialised area support the results of evaluations of user involvement initiatives more generally, although they also raise a number of questions. For example, at a workshop on user involvement in R\&D organised by the West Midlands Regional Office in December 2000, all speakers - including the director of the Consumers in NHS Research Support Unit-drew attention to the time consuming and resource intensive nature of involving users in research in a way that enabled them to make a meaningful contribution, so it is no surprise to see the authors make the same observation.

Inevitably this raises the question of whether there are the resources available to involve patients in the wide range of health service activities that are being opened up to them. At the very least it means that economies of scale should be sought and duplication avoided. In the area of guideline development it makes sense to put effort into the involvement of patients at the national level, and it is reassuring to see that the National Institute for Clinical Excellence is making good progress in this direction.

At the local level there will be more temptation to cut corners and fall into the trap of involving single patients in guideline groups. The finding by the authors that patients' contributions were small in the guideline groups where individuals were involved is fairly typical. The problems resulting from the inclusion of single lay members on committees of professionals have been reported in the literature many times before.

This apparent failure to contribute can be contrasted with the experience of the patient advocate who felt she had participated fully in the work of the group. As the head of a national voluntary organisation, the advocate would be seen by some as a "professional" patient and so less representative of "ordinary" patients. Certainly these views were found during a survey of public involvement in primary care. ${ }^{2}$ Yet having a knowledge of the particular perspectives that patients bring, identifying common issues, and developing the language to express them in interactions with health professionals is an acquired skill and one that has been found to be valuable on many occasions, including that described in the paper by van Wersch and Eccles. ${ }^{1}$

These findings show that, where group size makes it necessary that an individual is required to provide a lay perspective, this should be someone who has either been trained to speak on behalf of patients or is experienced at doing so. It also highlights the importance of investment in the training of lay representatives in the health service. ${ }^{3}$ Community health council chief officers have fulfilled this role, usually without training, for some time and it is not at all clear that the Patient Advocacy and Liaison Service proposed in the NHS Plan will improve this situation by the provision of training for those involved.

The group of patients drawn from the local branch of a national patient organisation also experienced no problems in expressing their views on the guidelines. They were particularly interested in the patient education and selfmanagement elements of the guidelines. This is noteworthy, given the government's interest in developing the concept of the "expert patient" announced in Our Healthier Nation and supported by a task force reporting to Professor Liam Donaldson, the Chief Medical Officer. Its work includes an investigation of ways in which patients can learn to deal more effectively with chronic conditions. There is evidence to show that the quality of patient education is questionable. Clark and Gong, ${ }^{4}$ for example, found that both patients and practitioners were being taught how to manage asthma in an inadequate way. One of their observations was that a patient is much more likely to be motivated to follow a practitioner's recommendations when the goal of management reflects the patient's own interests and concerns. In focusing on the areas of patient education and self-management, patients on guideline groups may be able to ensure that these are given sufficient attention.

The researchers came to the conclusion that, although the patients from the patient organisation were happy to take part, they were unable to grasp the importance of using scientific evidence to make health services more cost effective and this limited their contribution. When a similar group of patients was involved in four workshops that took them through a series of exercises to develop their understanding of how to use quality and cost criteria in assessments, however, their ability to contribute improved radically. This is consistent with other examples where lay people have been provided with relevant information ${ }^{5}$ and again draws attention to the importance of preparation and training.

Based on their findings, the authors recommend that appropriate support and training needs to be supplied to all members of guideline development groups to allow them to fulfil their role. With this in mind, it is time "health literacy" was introduced into the school curriculum so that in the future patients are ready to take on the mantle of co-producers of health and expert patients.

SHIRLEY MCIVER

Senior Fellow, Health Services Management Centre, University of Birmingham, Park House, 40 Edgbaston Park Road, Birmingham B15 2RT, UK

s.a.mciver@bham.ac.uk

1 Van Wersch A, Eccles M. Involvement of consumers in the development of evidence based clinical guidelines: practical experiences from the North of England evidence based guideline development programme. Quality Health Care 2001;10:10-7.

2 Barnes M, McIver S. Public participation in primary care. Research Report 36. Birmingham: Health Services Management Centre, University of Birmingham, 1999 .

3 Bradburn J, Fletcher G, Kennelly C. Voices in action: training and support for lay representatives in the health service. London: College of Health, 2000.

4 Clark NM, Gong M. Management of chronic disease by practitioners and patients: are we teaching the wrong things? BMF 2000;320:572-5.

5 McIver S. Healthy debate? An independent evaluation of citizens' juries in health settings. London: King's Fund, 1998.

6 Dolan P, Cookson R, Ferguson B. Effect of discussion and deliberation on the public's views of priority setting in health care: focus group study. $B M \mathcal{F}$ 1999;318:916-9. 